\title{
Karbon Keçe Elektrotun Alkali Ortamda Elektrokatalitik Hidrojen Çıkış Etkinliğinin Artırılması
}

\author{
Ali DÖNER ${ }^{* 1}$ \\ ${ }^{1}$ Şırnak Üniversitesi, Mühendislik Fakültesi, Enerji Sistemleri Mühendisliği Bölümü, Şırnak
}

Geliş tarihi: 09.07.2018 Kabul tarihi: 27.03.2019

$\ddot{\mathbf{O z}}$

Bu çalıșmada, karbon keçe üzerine bakır, kobalt, kobalt-bakır ve kobalt/platin elektrokimyasal olarak çöktürülmüştür. Hazırlanan elektrotların $1 \mathrm{M} \mathrm{KOH}$ çözeltisinde hidrojen gazı çıkışına katalitik etkisi katodik akım-potansiyel eğrileri, elektrokimyasal impedans spektroskopisi (EIS) ve elektroliz yöntemleri kullanılarak incelenmiş̧tir. Hazırlanan elektrotların zamanla kararlılık testi $1 \mathrm{M} \mathrm{KOH}$ çözeltisi içinde $2,2 \times 10^{-3} \mathrm{~A} \mathrm{~m}^{-2}$ lik akım yoğunluğunda araștırılmıștır. Elde edilen sonuçlara göre karbon keçe üzerine, kobalt, kobalt-bakır ve kobalt/platin çöktürülmesi elektrotun aşırı gerilimini düşürerek hidrojen gazı çıkışını arttırmaktadır. Elektrotun katalitik etkinliği çöktürülen metalin türüne bağlıdır. Katalitik etkinliğin artması keçe elektrotun büyük yüzey alanı ve kobalt ile platinin yüksek katalitik etkinliği ile açılanabilir.

Anahtar Kelimeler: Karbon keçe, Kobalt çöktürme, Elektrokataliz, Elektroliz, Hidrojen

\section{Enhancement of Electrocatalytic Hydrogen Evolution Activity of Carbon Felt in Alkaline Media}

\begin{abstract}
In this study, copper, cobalt, cobalt-copper and cobalt/platinum were electrochemically deposited over a carbon-felt electrode. The electrocatalytic activity of the prepared electrodes for the hydrogen gas evolution was studied in $1 \mathrm{M} \mathrm{KOH}$ solution using cathodic current-potential curves, electrochemical impedance spectroscopy (EIS) and electrolysis techniques. The long-term stability of the prepared electrodes was investigated at $2.2 \times 10^{-3} \mathrm{~A} \mathrm{~m}^{-2}$ in $1 \mathrm{M} \mathrm{KOH}$ solution. It was found that, the electrodeposition of the copper, cobalt, cobalt-copper and cobalt/platinum over the carbon-felt increases hydrogen gas evolution by decreasing discharge potential. The electrocatalytic activity of the electrode depends on the kind of metal. The electrocatalytic activity of deposited electrodes can explain by high surface area of the carbon felt and high catalytic activity of the cobalt and platinum.
\end{abstract}

Keywords: Carbon felt, Cobalt deposition, Electrocatalaysis, Electrolysis, Hydrogen

"Sorumlu yazar (Corresponding author): Ali DÖNER, ali@sirnak.edu.tr 


\section{GíRiş}

Dünya enerji kaynaklarının büyük çoğunluğu günümüzde fosil yakıtlardan sağlanmaktadır. Fosil yakıtların sürekli fiyatlarının artması, sınırlı kaynakları, insan sağlı̆̆ına ve çevreye olumsuz etkileri nedeniyle dünyadaki araştırmalar alternatif enerji kaynaklarına yönelmiştir. Bir yakıtın her yerde, örneğin sanayide, evlerde, taşıtlarda kullanılabilmesi büyük önem taşımaktadır. Diğer yakıtlarla karşılaştırıldığında, bunların birçoğunun ancak belirli uygulamalar için kullanılabildiğini görmekteyiz. Kömürü, otomobillerde veya uçaklarda kullanmak pratik açıdan uygun değildir. Hidrojen ise, hemen her yerde kolaylikla kullanılabilir. Geleceğin enerji kaynağı olarak gösterilen hidrojen, değişik yollarla elde edilebilir. Ancak en uygun yöntem elektroliz ile hidrojenin eldesidir. Bu yöntemde kullanılan elektrik enerjisi $\mathrm{H}_{2}$ birim fiyatını artırmaktadır. Ayrıca elektrot ve elektrolitlerden oluşan aşırı gerilimlerde ilave enerji gerektirdiğinden birim fiyatı daha da arttırmaktadır. Bu olumsuzlukların giderilmesi için uygun elektrot, çalışma ortamı ve katalizör araştırılmaktadır [1-5].

Karbon keçe oldukça büyük yüzey alanına sahip ancak hidrojen gazı oluşumu için yeterli etkinliğe sahip değildir. Karbon keçe üzerine hidrojen gazı çıkışı için yüksek katalitik etkinliğe sahip metaller çok az miktarlarda çöktürülerek arttırılabilir. Bu çalışmada karbon keçe üzerine farklı metaller elektrokimyasal olarak çöktürülmüş ve hazırlanan elektrotların $1 \mathrm{M} \mathrm{KOH}$ çözeltisinde hidrojen gazı çıkışına katalitik etkinlikleri incelenmiştir.

Suyun elektrolizinde hidrojen çıkışı reaksiyonu (HER) en önemli kritik basamak olup, pratik uygulamalar için yüksek etkinlikli ve ucuz elektrotlar kullanılmalıdır. Platin (Pt) HER'unu etkili bir şekilde katalizler ama Pt'in pahalı olması ve kısıtlı bulunması nedeniyle pek tercih edilmemektedir. Bunun yerine Xia ve arkadaşları [6] yaptıkları bir çalışmada çok az miktarda Pt-Au nano taneciklerini grafin üzerine çöktürmüşler ve HER performansını araştırmışlardır. Sonuçlara göre tek tabaka Pt-Au, ikili ve üçlü tabakaya göre elektronik özellikten dolayı çok iyi HER aktivitesi göstermiştir. Badawy ve arkadaşları [7] yaptıkları çalışmada ise $\mathrm{Ni}$ ve NiCo kaplamasını elektrokimyasal çöktürme yöntemi ile bakır elektrot üzerinde oluşturmuş ve HER özelliklerini araştırmışlardır. Elde edilen sonuçlara göre $\mathrm{Ni}$ matriksi içine kobalt iyonlarının yerleştirilmesi ve kobalt oranın $\% 50$ seviyesine kadar arttırılması Hidrojen aşırı gerilimini düşürmüştür. Bunun sebebini ise iki metalin kaplamada meydana getirdiği sinerjistik etkinin ortaya çıkmasına dayandırmışlardır. Solmaz [8], NiIr kaplamasını Nikel ile modifiye edilmiş karbon keçe üzerine elektrokimyasal çöktürme yöntemi ile hazırlamıştır. Hazırlanan kaplamanın HER etkinliği alkali ortamda araştırılmıştır. Elde edilen sonuçlara göre NiIr kaplamasının yüksek HER aktifliği karbon keçenin sahip olduğu geniş yüzey alanına, ince yüzey yapısına, çok fazla katalitik aktif merkezlerin varlığına ve metal iyonlarının birbiriyle etkileşimine bağlamıştır. Patel ve ark. [9] kobalt-borit (Co-B) katalizörünü sentezleyerek geniş bir $\mathrm{pH}$ aralığındaki çözeltilerde HER kinetiğini araştırmışlardır. Nötral çözeltide elde edilen akım değerlerinin yüksek olduğunu ve düşük aşırı gerilimler oluştuğunu belirterek mekanizmanın Volmer-Heyrovsky basamağ üzerinden yürüdüğünü açıklamışlardır. X-ışını fotoelektron spektroskopisi (XPS) analizlerine göre bordan kobalta elektronik transfer sayesinde büyük yüzey alanlı kobalt bölgelerinin oluştuğunu göstermişlerdir. Casciano ve arkadaşları [10] yaptıkları çalışmada alkali ortamda, Co-Mo kaplamasının HER etkinliği üzerine çöktürme akım yoğunluğunun, çöktürme banyoların $\mathrm{pH}$ değerinin ve yüzey porözite faktörünün etkilerini araştırmışlardır. $\mathrm{Bu}$ amaçla seçilen akım yoğunlukları ve $\mathrm{pH}$ değerleri sirasiyla 30, 45, $60 \mathrm{~mA} \mathrm{~cm}^{-2}$ ve 6,7 ve 8 'dir. Elde edilen bulgulara göre kaplamalar çatlak ve amorf yapıya sahip, pH'ın HER etkinliğine pozitif etki ettiği ve yüksek akım yoğunluklarının HER performansını katalizlediğini bulmuşlarıdır. Ayrıca HER kinetiğinde Volmer basamağının hız belirleyici basamak olduğunu ve aktivasyon enerjisinin $18,40 \mathrm{~kJ} / \mathrm{mol}$ olduğunu belirtmişlerdir.

\section{MATERYAL VE METOT}

Karbon keçe (C) 0,006 m x 0,02 m x 0,01 m boyutlarında kesilerek elektrot yapımında 
kullanılmıştır. Kullanılan keçenin yüzey alanı

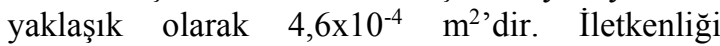
sağlamak için $0,2 \mathrm{~m}$ uzunluğunda $0,001 \mathrm{~m}$ çapındaki bakır tel karbon keçeye tutturularak çalışma elektrotları hazırlanmıştır. Hazırlanan karbon keçe elektrotlar çöktürme banyolarına daldırılarak 30 dakika boyunca çöktürme işlemi yapılmıştır. Kobalt çöktürme banyosu: \%30 $\mathrm{CoSO}_{4} .7 \mathrm{H}_{2} \mathrm{O}, \% 1 \quad \mathrm{CoCl}_{2} .6 \mathrm{H}_{2} \mathrm{O}, \% 1,25 \quad \mathrm{H}_{3} \mathrm{BO}_{3}$. Bakır çöktürme banyosu: \%26,67 $\mathrm{CuSO}_{4} .5 \mathrm{H}_{2} \mathrm{O}$, $\% 1,25 \quad \mathrm{H}_{3} \mathrm{BO}_{3}$. Kobalt-Bakır çöktürme banyosu: $0,044 \mathrm{~mol} \mathrm{CoSO}_{4} \cdot 7 \mathrm{H}_{2} \mathrm{O}, \quad 5,33 \times 10^{-4} \mathrm{~mol}$ $\mathrm{CuSO}_{4} .5 \mathrm{H}_{2} \mathrm{O}, \% 5$ Sodyum sitrat ve $\% 1,25 \mathrm{H}_{3} \mathrm{BO}_{3}$. Platin çöktürme banyosu: $5,66 \times 10^{-4} \mathrm{~g} \mathrm{Pt} / 0,05 \mathrm{~L}$ çözelti olacak şekilde $\mathrm{K}_{2} \mathrm{PtCl}_{4}$ ilave edilerek hazırlanmıştır. Destek elektrolit olarak $\mathrm{KCl}$ kullanılmıştır. Kaplamalar, elektroliz sistemine sabit 0,02 A katodik akım 30 dakika boyunca uygulanarak Poansiyostat\&Galvanostat cihazı kullanılarak yapılmıştır. $\mathrm{Bu}$ amaçla bakır kaplamalarda bakır anot, kobalt, kobalt-bakır ve platin kaplamalarda platin anot, çalışma elektrotları katot ve $\mathrm{Ag} / \mathrm{AgCl}, \mathrm{Cl}^{-}$referans elektrot olarak kullanılmıştır. Kaplamalar oda sıcaklığında, banyo çözeltileri karıştırılarak yapılmıştır. Katodik akım-potansiyel eğrileri ve EIS ölçümleri CHI 660c elektrokimyasal analizör cihazı ile üç elektrot tekniği kullanılarak elde edilmiştir. Karşı elektrot olarak Pt ve referans elektrot olarak $\mathrm{Ag}, \mathrm{AgCl} / \mathrm{Cl}^{-}$ elektrotu kullanılmıştır. Katodik akım-potansiyel eğrileri -1,80 V ile sıfır akım potansiyeli aralığında $0,005 \mathrm{~V} \mathrm{~s}^{-1}$ tarama hızı ile elde edilmiștir. Katodik akım-potansiyel eğrilerinden belirlenen ve hidrojen gazı çıkışının gerçekleştiği değişik aşırı gerilimlerde EIS ölçümleri yapılmıştır. EIS ölçümleri $10^{5} \mathrm{~Hz}$ ile $0,003 \mathrm{~Hz}$ frekans aralığında sisteme $0,005 \mathrm{~V}$ genlik uygulanarak elde edilmiştir. Elektroliz sisteminde platin anot ve çalışma elektrotları katot olacak şekilde sisteme sabit 3,0 V potansiyel 30 dakika boyunca uygulanmış ve çıkan hidrojen gazı, içerisine çalışma çözeltisi doldurulmuş ve katot üzerine ters çevrilerek yerleştirilmiş bir büret içerisinde toplanmıştır. Basınç düzeltmesi literatürde [11] verildiği gibi yapılarak saf hidrojen hacmi belirlenmiştir. Etkinliği yüksek $\mathrm{C} / \mathrm{Co}$ ve $\mathrm{C}-\mathrm{Co} / \mathrm{Pt}$ elektrotların $1 \mathrm{M} \mathrm{KOH}$ çözeltisindeki kararlılı̆̆ oda sıcaklığında 120 saat boyunca test edilmiştir. $\mathrm{Bu}$ amaçla çalışma elektrodu katot ve $\mathrm{Pt}$ anot olacak şekilde iki elektrot tekniği kullanılarak elektoliz sistemine sabit $2,2 \times 10^{-3} \mathrm{~A} \mathrm{~m}^{-2}$ katodik akım 120 saat boyunca uygulanmıştır. 24 saat ara ile elektroliz durdurularak katodik akım-potansiyel eğrileri ve EIS teknikleri kullanılarak elektrotun katalitik etkinliğinin zamanla değişimi belirlenmiştir.

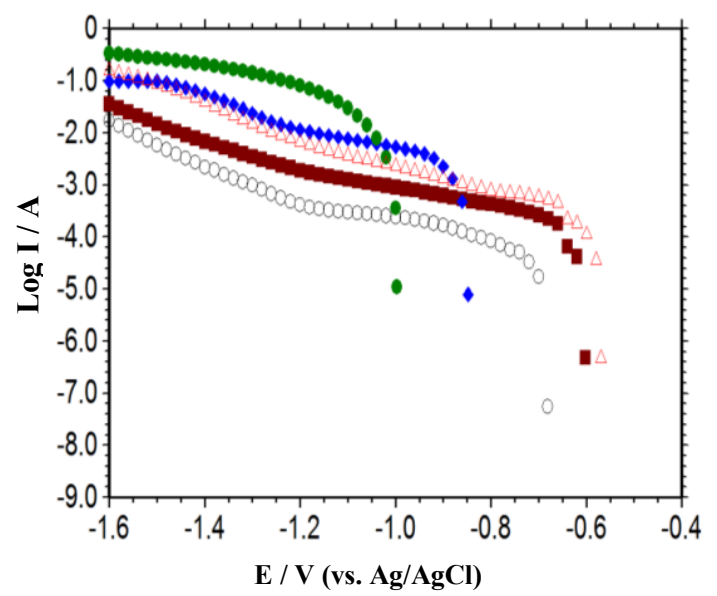

Şekil 1. $1 \mathrm{M} \mathrm{KOH}$ çözeltisi içerisinde C-Keçe (O), $\mathrm{C}-\mathrm{Cu}(\boldsymbol{\bullet}), \mathrm{C}-\mathrm{CoCu}(\Delta), \mathrm{C}-\mathrm{Co}(\diamond)$ ve $\mathrm{C}-\mathrm{Co} / \mathrm{Pt}(\bullet)$ elektrotların katodik akımpotansiyel eğrileri

\section{BULGULAR VE TARTIŞMA}

$1 \mathrm{M} \mathrm{KOH}$ çözeltisi içerisinde alınan C-Keçe, $\mathrm{C}-\mathrm{Cu}, \mathrm{C}-\mathrm{CoCu}, \mathrm{C}-\mathrm{Co}$ ve $\mathrm{C}-\mathrm{Co} / \mathrm{Pt}$ elektrotların katodik akım-potansiyel eğrileri Şekil 1'de verilmektedir. Saf keçe elektrot için elde edilen eğri incelendiğinde sıfır akım potansiyelinden itibaren daha katodik potansiyele gidildiğinde yaklaşık -0,9 V ile $-1,19 \mathrm{~V}$ potansiyel aralığında akım fazla değişmemektedir. $\mathrm{Bu}$ potansiyel aralığında okunan akım değeri oksijen sınır akımına karşılık gelmektedir. Karbon keçe elektrotta -1,2 V'tan itibaren hidrojen indirgenmesi ile birlikte akım artışı olmaktadır. 30 dakika bakır, kobalt-bakır ve kobalt çöktürülmüş karbon keçe elektrotlarda ise $\sim-1,15$, $-1,16$ ve $-1,17$ V'tan itibaren akım artmaktadır. 
Çizelge 1. Çalışma elektrotları için elde edilen katodik akım-potansiyel eğrilerinden değişik aşırı gerilimlerde belirlenen akım yoğunluğu değerleri

\begin{tabular}{|c|c|c|c|}
\hline Elektrot & \multicolumn{3}{|c|}{$\mathrm{I}\left(\mathrm{A} \mathrm{m}^{-2}\right)$} \\
\hline & $-1,2 \mathrm{~V}$ & $-1,3 \mathrm{~V}$ & $-1,4 \mathrm{~V}$ \\
\hline $\mathrm{C}-\mathrm{Keçe}$ & 0,09 & 0,2 & 0,5 \\
\hline $\mathrm{C}-\mathrm{Cu}$ & 0,4 & 0,8 & 1,5 \\
\hline $\mathrm{C}-\mathrm{CoCu}$ & 1,5 & 3,1 & 8,6 \\
\hline $\mathrm{C}-\mathrm{Co}$ & 2,5 & 5,2 & 12,3 \\
\hline $\mathrm{C}-\mathrm{Co} / \mathrm{Pt}$ & 17,7 & 30,5 & 43,9 \\
\hline
\end{tabular}

Kobalt/platin çöktürülmüş karbon keçe elektrotta oksijen sınır akımının oluşmadığı ve her bölgede hidrojen indirgenmesinin olduğu görülmektedir. $\mathrm{Bu}$ sonuç keçe üzerine kobalt/platin çöktürülmesi ile elektrotun aşırı gerilimini düşürerek elektrotun etkinliğini arttırdığını göstermektedir. Elde edilen katodik akım-potansiyel eğrilerinden hidrojen gazı çıkışının gerçekleştiği değişik potansiyellerde akım değerleri belirlenmiş ve Çizelge 1 'de verilmiştir. Şekil 1 ve Çizelge 1'den de görüldüğü gibi kobalt çöktürülmüş karbon keçe elektrotun yüzeyine az miktarda platinin çöktürülmesi hidrojen gazı çıkışı için elektrotun katalitik etkisini arttırmaktadır. Kobalt ve kobalt/platin çöktürülmüs elektrotların yüksek etkinliği karbon keçenin büyük yüzey alanı ve platin ve kobaltın yüksek katalitik etkisi ile açıklanabilir $[12,13]$.

$\mathrm{C}, \mathrm{C}-\mathrm{Cu}, \mathrm{C}-\mathrm{CoCu}$ ve $\mathrm{C} / \mathrm{Co}$ elektrotların $1 \mathrm{M} \mathrm{KOH}$ çözeltisinde hidrojen gazının oluştuğu $-1,2 \mathrm{~V}$ potansiyelde elde edilen Nyquist eğrileri Şekil 2'de verilmiștir. C-Co/Pt elektrotun șekli ise Şekil 2'nin iç kısmında verilmiştir. Şekil 2'den de görüldüğü gibi bütün çalışma elektrotları için yarım daireden sapan basık eliptik luplar oluşmuştur. Elde edilen yarım dairelerin yarıçapı genellikle yük transfer direnci (Rt) olarak kabul edilmektedir [14,15]. Nyquist eğrilerinden belirlenen yük transfer dirençleri Çizelge 2'de verilmektedir. Elde edilen değerler incelendiğinde aynı potansiyelde kobalt/platin çöktürülmüş elektrotta yük transfer direncinin diğer metal çöktürülmüş elektrotlara göre önemli ölçüde düştüğü görülmektedir. $\mathrm{Bu}$ sonuçlar karbon keçe üzerine kobalt/platin çöktürülmesi ile sistemdeki aşırı gerilimlerin düştüğü ve hidrojen gazı oluşumu sırasında daha düşük bir dirençle karşılaşıldığını göstermektedir.

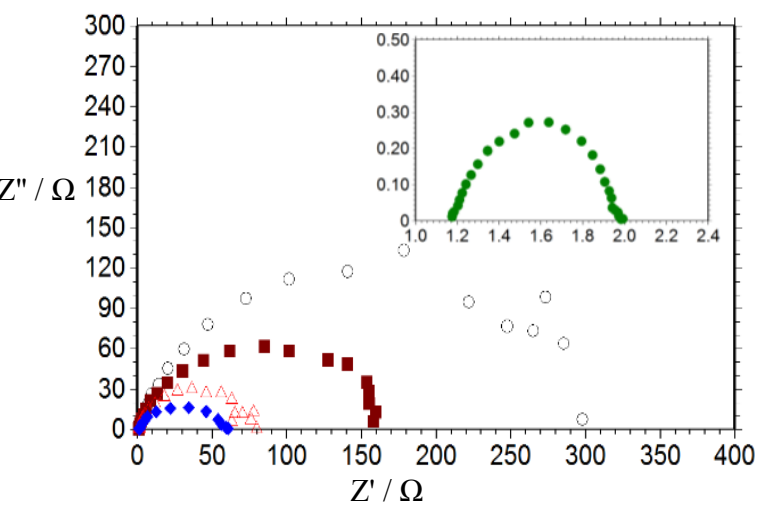

Şekil 2. $1 \mathrm{M}$ KOH çözeltisi içerisinde C-Keçe (o), $\mathrm{C}-\mathrm{Cu}(\mathbf{\square}), \mathrm{C}-\mathrm{CoCu}(\Delta), \mathrm{C}-\mathrm{Co}(\diamond)$ ve $\mathrm{C}-\mathrm{Co} / \mathrm{Pt}(\bullet)$ elektrotların $-1,2 \quad \mathrm{~V}$ aşırı gerilimindeki Nyquist eğrileri

Çizelge 2. Çalışma elektrotları için elde edilen nyquist eğrilerinden belirlenen polarizasyon dirençleri

\begin{tabular}{|c|c|c|c|}
\hline Elektrot & \multicolumn{3}{|c|}{$\mathrm{Rt} / \Omega$} \\
\hline & $-1,2 \mathrm{~V}$ & $-1,3 \mathrm{~V}$ & $-1,4 \mathrm{~V}$ \\
\hline $\mathrm{C}-\mathrm{Keçe}$ & 327,0 & 69,6 & 23,5 \\
\hline $\mathrm{C}-\mathrm{Cu}$ & 176,3 & 40,4 & 17,3 \\
\hline $\mathrm{C}-\mathrm{CoCu}$ & 71,6 & 18,7 & 4,8 \\
\hline $\mathrm{C}-\mathrm{Co}$ & 59,2 & 7,9 & 2,4 \\
\hline $\mathrm{C}-\mathrm{Co} / \mathrm{Pt}$ & 0,9 & 0,6 & 0,3 \\
\hline
\end{tabular}

Nyquist eğrilerinde sadece bir lupun oluşması hidrojen çıkış reaksiyonunun aktivasyon kontrollü olduğunu göstermektedir [14,15]. Oluşan lupun yarım daireden sapması elektrot yüzeyinin gözenekli olduğunu göstermektedir [16].

Çalışma elektrotları katot ve platin anot olmak üzere iki elektrot tekniği kullanılarak elektroliz sistemine sabit $3,0 \mathrm{~V}$ potansiyel uygulanarak katotta oluşan hidrojen gazının hacmi ölçülmüsstür. 30 dakika sonunda çalışma elektrotlarında ölçülen hidrojen gazı hacimleri Şekil 3'te verilmiştir. Ölçülen hidrojen gazı hacimleri incelendiğinde karbon keçe yüzeyine bakır, kobalt-bakır, kobalt ve kobalt/platin çöktürüldüğünde elde edilen hidrojen gazı hacminin arttığı görülmektedir. 


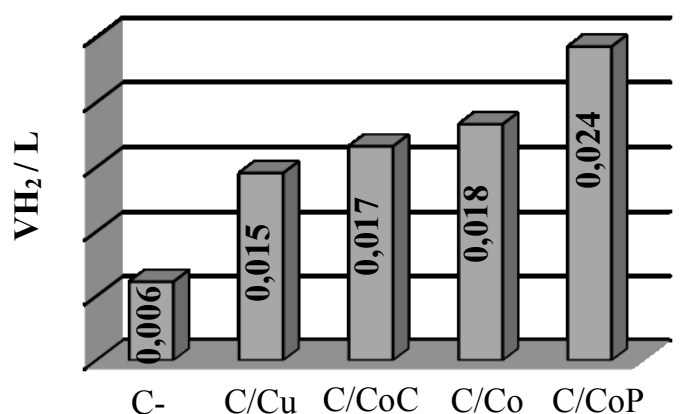

Şekil 3. $1 \mathrm{M}$ KOH çözeltisi içerisinde çalışma elektrotlarında 30 dakika sonunda açığa çıkan hidrojen gazı hacimleri

Elektroliz sisteminde kullanılan elektrotların yüksek katalitik etkinliğe sahip olması gerekmektedir. Bununla birlikte elektrotların katalitik etkinliğinin zamanla kararlı olması ve azalmaması gerekmektedir. $\mathrm{Bu}$ amaçla katalitik etkinliği yüksek olan $\mathrm{C}-\mathrm{Co}$ ve $\mathrm{C}-\mathrm{Co} / \mathrm{Pt}$ elektrotların $1 \mathrm{M} \mathrm{KOH}$ çözeltisindeki kararlılığı oda sıcaklığında 120 saat boyunca test edilmiştir. Şekil 4 ve 5'de C-Co ve C-Co/Pt elektrotların 24 saat aralıklar ile alınan katodik akım-potansiyel eğrileri görülmektedir. Şekil 4 incelendiğinde C-Co elektrotu için katalitik etkinliğinin elektroliz süresinin artması ile hemen hemen sabit kaldığ 1 görülmektedir. Sabit kalmasının nedeni çalışılan elektrotun o ortamda kararlı bir yapıya sahip olduğunu gösterir. Elektrotun açık devre potansiyeli elektroliz süresinin artması ile daha pozitif potansiyele kaymaktadır. Potansiyelin daha pozitif değerlere kayması elektrotun yüzeyinin değiştiğini göstermektedir [17].

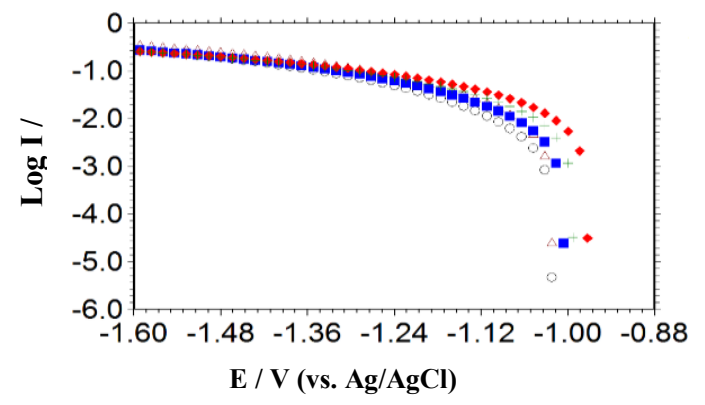

Şekil 4. $1 \mathrm{M}$ KOH çözeltisi içerisinde C-Co elektrotun 24(०), 48( $\Delta), 72(\mathbf{\square}), 96(+)$ ve 120( $)$. saatteki katodik akım-potansiyel eğrileri

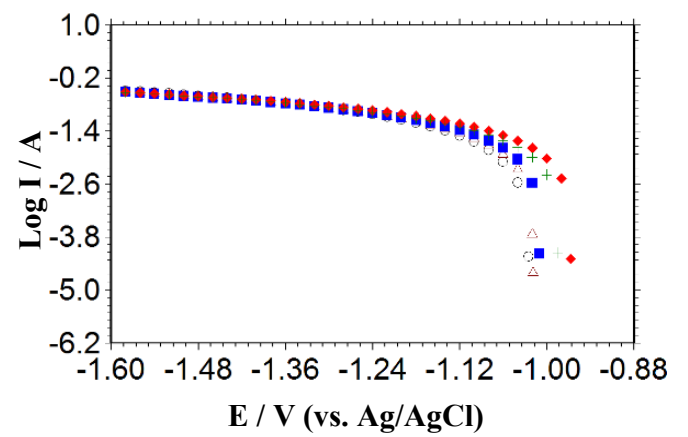

Şekil 5. $1 \mathrm{M}$ KOH çözeltisi içerisinde $\mathrm{C}-\mathrm{Co} / \mathrm{Pt}$

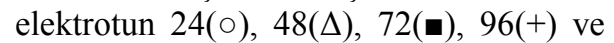
$120(\diamond)$. saatteki katodik akım-potansiyel eğrileri

Şekil 5'e bakıldığg zaman, C-Co/Pt elektrotu için akım değerlerinin zamanla arttığı görülmektedir. Akım değerlerinin artması elektrotun gözeneklerindeki korozyon ürünlerinin elektroliz sırasında çıkan hidrojen gazı ile gözeneklerden uzaklaşması sonucu elektrot yüzeyindeki gözeneklerin açılması ve elektrotun yüzey alanının artması ile açıklanabilir [18]. Katodik akım-potansiyel eğrilerinden değişik aşırı gerilimlerde belirlenen akım yoğunlukları Çizelge 3'te verilmektedir.

Çizelge 3. $1 \mathrm{M} \mathrm{KOH}$ çözeltisi içerisinde C-Co ve C-Co/Pt elektrotların 24, 48, 72, 96 ve 120. saatteki katodik akım-potansiyel eğrilerinden değişik aşırı gerilimlerde belirlenen akım yoğunlukları

\begin{tabular}{|c|c|c|c|c|}
\hline Elektrot & \multicolumn{4}{|c|}{$\mathrm{I}\left(\mathrm{A} \mathrm{m}^{-2}\right) \times 10^{-3}$} \\
\hline \multirow{6}{*}{$\Theta^{0}$} & $\begin{array}{l}\text { Elektroliz } \\
\text { süresi }\end{array}$ & $-1,2 \mathrm{~V}$ & $-1,3 \mathrm{~V}$ & $-1,4 \mathrm{~V}$ \\
\hline & 24 & 4,2 & 11,8 & 23,7 \\
\hline & 48 & 12,1 & 24,1 & 39,2 \\
\hline & 72 & 9,8 & 19,7 & 32,0 \\
\hline & 96 & 12,4 & 21,8 & 32,8 \\
\hline & 120 & 14,8 & 24,0 & 34,4 \\
\hline \multirow{5}{*}{$\underbrace{+}_{0}$} & 24 & 18,1 & 32,3 & 47,7 \\
\hline & 48 & 18,7 & 32,0 & 46,3 \\
\hline & 72 & 19,9 & 32,6 & 46,3 \\
\hline & 96 & 23,8 & 36,0 & 48,7 \\
\hline & 120 & 21,3 & 33,5 & 46,6 \\
\hline
\end{tabular}


Çizelge 3'e bakıldığı zaman C-Co elektrotu için 24 . saatteki akım yoğunluğu $-1,2 \mathrm{~V}$ 'ta $4,2 \times 10^{-3} \mathrm{~A} \mathrm{~m}^{-2}$, 120. saatin sonunda $14,8 \times 10^{-3} \mathrm{~A} \mathrm{~m}^{-2}$, bu değerler $\mathrm{C}$ $\mathrm{Co} / \mathrm{Pt}$ elektrotu için 24. saatin sonunda $18,1 \times 10^{-3} \quad \mathrm{~A}^{-2}$ ve 120 . saatin sonunda $21,3 \times 10^{-3} \quad \mathrm{~A} \mathrm{~m}^{-2}$ olduğu görülmektedir. Akım yoğunluğu değişimi $\mathrm{C}-\mathrm{Co} / \mathrm{Pt}$ elektrotta daha az olduğu için bu elektrotta katalitik etkinliğin hemen hemen değişmediği ve elektrotun zamanla oldukça kararlı olduğu söylenebilir.

C-Co ve C-Co/Pt elektrotların 24, 48, 72, 96 ve 120 saat elektroliz edildikten sonra $-1,2 \mathrm{~V}$ aşırı gerilimde elde edilen Nyquist eğrileri Şekil 6 ve 7 'de verilmiştir. Şekillerden de görüldüğü gibi eğriler gözenekli elektrotların tipik şekillerini oluşturmaktadır [19].

Bütün elektrotlarda yüksek frekans bölgesinde başlayan ve düşük frekans bölgesinde kapanan basık bir lup oluşmaktadır. Oluşan eğrilerin yarım daireden sapması yani basık olması elektrotun yüzeyinin gözenekli olduğunu göstermektedir $[13,18]$.

Her iki elektrot içinde elektroliz süresinin artması ile direnç değerlerinde önemli bir değişiklik olmamakta bu da elektrotların zamanla kararlı bir yapıya sahip olduğunu göstermektedir.

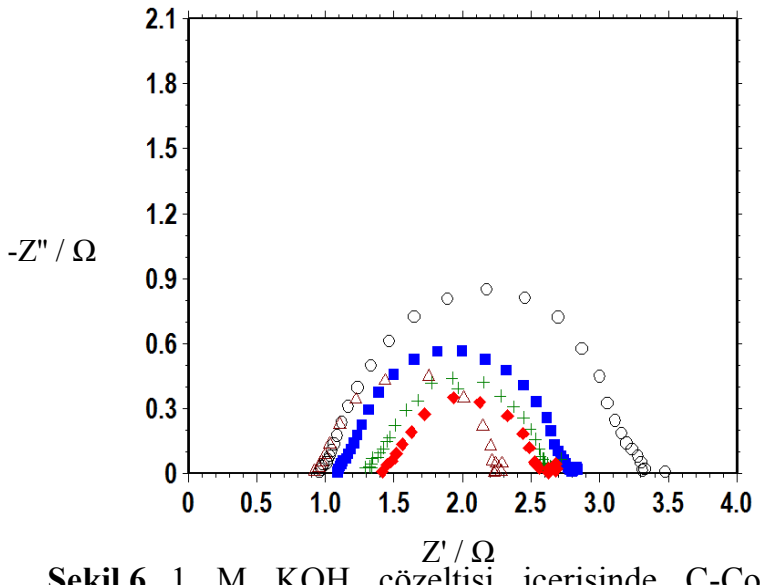

Şekil 6. $1 \mathrm{M} \quad \mathrm{KOH}$ çözeltisi içerisinde $\mathrm{C}-\mathrm{Co}$

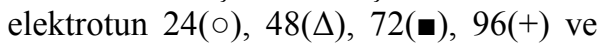
120(৫). saatteki $-1,2 \mathrm{~V}$ aşırı gerilimdeki nyquist eğrileri

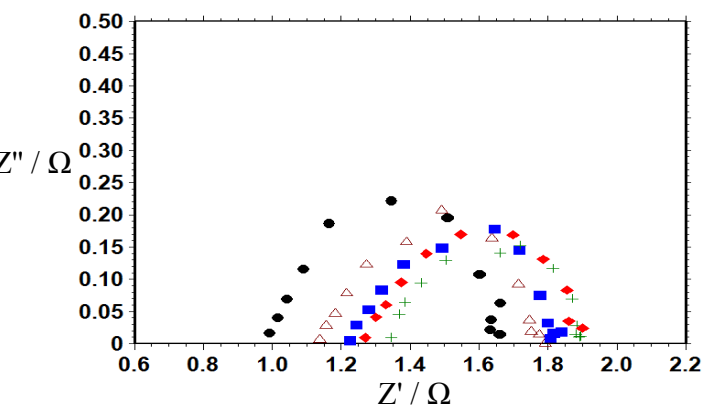

Şekil 7. $1 \mathrm{M} \mathrm{KOH}$ çözeltisi içerisinde $\mathrm{C}-\mathrm{Co} / \mathrm{Pt}$ Elektrotun $24(\bullet), 48(\Delta), 72(\bullet), 96(+)$ ve 120(»). saatteki $-1,2 \mathrm{~V}$ aşırı gerilimdeki nyquist eğrileri

Katodik akım-potansiyel eğrilerinden belirlenen akım yoğunlukları ile Nyquist eğrilerinden belirlenen direnç değerleri Çizelge 4'de verilmektedir. Elde edilen sonuçlara göre karbon keçe üzerine kobalt, kobalt-platin çöktürülmesi elektrotun aşırı gerilimini düşürerek elektrotun etkinliğini önemli ölçüde arttırmaktadır. Elektrotun katalitik etkinliği çöktürülen metalin türüne bağl1 olarak değişmektedir. Aynı potansiyelde en yüksek akım ve en düşük yük transfer direnci $\mathrm{C}-\mathrm{Co} / \mathrm{Pt}$ elektrotta elde edilmiştir. C-Co/Pt elektrotun yüksek katalitik etkinliği keçe elektrotun büyük yüzey alanı ve çöktürmeye eklenen $\mathrm{Pt}$ 'in yüksek katalitik etkinliği ile açıklanmıştır.

Çizelge 4. $1 \mathrm{M} \mathrm{KOH}$ çözeltisi içerisinde C-Co ve C-Co/Pt elektrotların 24, 48, 72, 96 ve 120. saatteki değişik aşırı gerilimlerde nyquist eğrilerinden belirlenen direnç değerleri

\begin{tabular}{|c|c|c|c|c|}
\hline Elektrot & \multicolumn{4}{|c|}{$\mathrm{Rt} / \Omega$} \\
\hline \multirow{6}{*}{ ن } & $\begin{array}{c}\text { Elektroliz } \\
\text { süresi }\end{array}$ & $-1,2 \mathrm{~V}$ & $-1,3 \mathrm{~V}$ & $-1,4 \mathrm{~V}$ \\
\hline & 24 & 2,37 & 1,10 & 0,55 \\
\hline & 48 & 1,35 & 0,71 & 0,49 \\
\hline & 72 & 1,66 & 0,92 & 0,67 \\
\hline & 96 & 1,28 & 0,93 & 0,64 \\
\hline & 120 & 1,15 & 0,83 & 0,64 \\
\hline \multirow{5}{*}{ 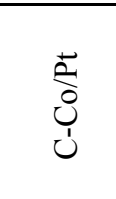 } & 24 & 0,67 & 0,51 & 0,39 \\
\hline & 48 & 0,65 & 0,52 & 0,39 \\
\hline & 72 & 0,60 & 0,53 & 0,45 \\
\hline & 96 & 0,60 & 0,52 & 0,44 \\
\hline & 120 & 0,64 & 0,56 & 0,47 \\
\hline
\end{tabular}


Katalitik etkinliği yüksek C-Co ve C-Co/Pt elektrotların zamanla kararlılık testi yapılmış ve çalışılan ortamda oldukça kararlı olduğu saptanmıştır. $\mathrm{Bu}$ sonuçlara göre $\mathrm{C}$ - $\mathrm{Co} / \mathrm{Pt}$ elektrot elektroliz sisteminde kullanıldığı zaman hidrojen gazı oluşumu sırasında elektroliz sisteminde daha az elektrik enerjisi harcanacaktır.

\section{TEŞEKKÜR}

Deneysel çalışmalar Çukurova Üniversitesi FenEdebiyat Fakültesi Kimya Bölümü Fizikokimya Araştırma Laboratuvarında yapılmıştır. Bu nedenle deneysel ölçümler için Çukurova Üniversitesine teşekkür ederiz.

\section{KAYNAKLAR}

1. Wu, J.K., 1992. Electrochemical Method for Studying Hydrogen in Iron, Nickel and Palladium, International Journal of Hydrogen Energy, 17, 917-921.

2. Petrii, O.A., Tsirlia, G.A., 1994. Electrocatalytic Activity Prediction for Hydrogen Electrode Reaction: Intuition, Art, Science, Electrochimica Acta, 39, 1739-1747.

3. Hu, W., Cao, X., Wang, F., Zhag, Y., 1997. A Novel Cathode for Alkaline Water Electrolysis, International Journal of Hydrogen Energy, 22, 621-623.

4. Kawashima, A., Akiyama, E., Habazaki, H., Hashimoto, K., 1997. Characterization of Sputter-deposited Ni-Mo and Ni-W Alloy Electrocatalysts for Hydrogen Evolution in Alkaline Solution, Material Science and Engineering, A226-228, 905-909.

5. Ananth, M.V., Parthasaradhy, N.V., 1997. Hydrogen Evolution Characteristics of Electrodeposited Ni-Zn-Fe Coatings in Alkaline Solutions, International Journal of Hydrogen Energy, 22, 747-751.

6. Yi, S., Ting-Ting, Z., Yue, Z., Wei-Xuan, X., Dong-Rui, Y., Feng-Bin, W., Xing-Hua, X., 2018. Atomic Level Tailoring of the Electrocatalytic Activity of Au-Pt Core-shell Nanoparticles with Controllable Pt Layers Toward Hydrogen Evolution Reaction, Journal of Electroanalytical Chemistry, 819, 442-446.

7. Badawy, W.A., Nady, H., Negem, M., 2014. Cathodic Hydrogen Evolution in Acidic
Solutions Using Electrodeposited NanoCrystalline Ni-Co Cathodes, International Journal of Hydrogen Energy, 39, 10824-10832.

8. Solmaz, R., 2013. Electrochemical Preparation and Characterization of $\mathrm{C} / \mathrm{Ni}-\mathrm{NiIr}$ Composite Electrodes as Novel Cathode Materials for Alkaline Water Electrolysis, International Journal of Hydrogen Energy, 39, 2251-2256.

9. Gupta, S., Patel, N., Miotello, A., Kothari, D. C., 2015. Cobalt-Boride: An Efficient and Robust Electrocatalyst for Hydrogen Evolution Reaction, Journal of Power Sources, 279, 620-625.

10. Casciano, P.N.S., Benevides, R.L., Santana, R.A.C., Correia, A.N., Lima-Neto, P., 2017. Factorial Design in the Electrodeposition of Co-Mo Coatings and Their Evaluations for Hydrogen Evolution Reaction, Journal of Alloys and Compounds, 723, 164-171.

11. Kardaş, G., Yazıcı, B., Erbil, M., 2003. Effect of Some Primary Alcohols on Hydrogen Yield on Platinum Cathode in Chloride Solution, International Journal of Hydrogen Energy, 28, 1213-1218.

12. Solmaz, R., Döner, A., Şahin, İ., Kardaş, G., Yazıc1, B., Erbil, M., 2008. Electrochemical Deposition, Characterization and Application of NiCo Coatings as Effective Cathode Materials for Hydrogen Production, BIES'08Blacksea Environmental Symposium, Giresun, Turkey.

13. Solmaz, R., Kardaş, G., 2007. Hydrogen Evolution and Corrosion Performance of NiZn Coatings. Energy Conversion and Management, 48, 583-591.

14. Rosalbino, F., Maccio, D., Angelini, E., Saccone, A., Delfino, S., 2005. Electrocatalytic Properties of $\mathrm{Fe}-\mathrm{R}(\mathrm{R}=$ rare earth metal $)$ Crystalline Alloys as Hydrogen Electrodes in Alkaline Water Electrolysis, Journal of Alloys and Compounds, 403, 275-282.

15. Navvaro-Flores, E., Chong, Z., Omanovic, S., 2005. Charactaerization of $\mathrm{Ni}, \mathrm{NiMo}, \mathrm{NiW}$ and $\mathrm{NiFe}$ Electroactive Coatings as Electrocatalysts for Hydrogen Evolution in an Acidic Medium, Journal of Molecular Catalysis A: Chemical, 226, 179-197.

16. Elumalai, P., Vasan, H.N., Munichandraiah, N., Shivashankar, S.A., 2002. Kinetics of 
Hydrogen Evolution on Submicron Size Co, $\mathrm{Ni}, \mathrm{Pd}$ and Co-Ni Alloy Powder Electrodes by D.C. Polarization and a.c. Impedance Studies, Journal of Applied Electrochemistry, 32, 1005-1010.

17. Solmaz, R., Döner, A., Şahin, I., Yüce, A.O., Kardaş, G., Yazıcı, B., Erbil, M., 2009. The Stability of NiCoZn Electrocatalyst for Hydrogen Evolution Activity in Alkaline Solution During Long-term Electrolysis, International Journal of Hydrogen Energy, 34, $7910-7918$.

18. Solmaz, R., Döner, A., Kardaş, G., 2009. The Stability of Hydrogen Evolution Activity and Corrosion Behavior of $\mathrm{NiCu}$ Coatings with Long-term Electrolysis in Alkaline Solution, International Journal of Hydrogen Energy, 34, 2089-2094.

19. Birry, L., Lasia, A., 2004. Studies of the Hydrogen Evolution Reaction on Raney Nickel-molybdenum Electrodes. Journal of Applied Electrochemistry, 34, 735-749. 